\title{
Photovoltaic properties of bisazomethine dye thin films
}

Takuya Hosokai $^{\text {a, f, }{ }^{*} \text {, Tetsuya Aoyama }}{ }^{\mathrm{b}}$, Takashi Kobayashi ${ }^{\mathrm{c}}$, Aiko Nakao ${ }^{\mathrm{d}}$ and Shinya Matsumoto ${ }^{\mathrm{a}, \mathrm{e}}$

${ }^{a}$ Graduate School of Environment and Information Sciences, Yokohama National University, 79-2

Tokiwadai, Hodogaya-ku, Yokohama 240-8501, Japan

${ }^{\mathrm{b}}$ Supramolecular Science Laboratory, RIKEN, 2-1 Hirosawa, Wako 351-0198, Japan

${ }^{\mathrm{c}}$ Faculty of Engineering, Osaka Prefecture University, 1-1 Gakuen-cho, Sakai 599-8531, Japan

${ }^{\mathrm{d}}$ Advanced Technology Support Division, RIKEN, 2-1 Hirosawa, Wako 351-0198, Japan

${ }^{\mathrm{e}}$ Faculty of Education and Human Sciences, Yokohama National University, 79-2 Tokiwadai, Hodogaya-ku, Yokohama 240-8501, Japan

Present address:

${ }^{\mathrm{f}}$ Institut für Angewandte Physik, Universität Tübingen, Auf der Morgenstelle 10, 72076 Tübingen, Germany

Photovoltaic properties of bisazomethine dye (DE2) in vacuum-deposited thin films were examined for use in Schottky-type photovoltaic cells (ITO/DE2/Al). We measured dark and photo current-voltage characteristics and determined photocurrent action spectra. We confirmed the p-type semiconductor property of DE2 thin films evidenced by the formation of the Schottky barrier at the DE2/Al interface. We further found a relatively high incident photon to electron conversion efficiency comparable to that of metal phthalocyanine thin films in devices having the same structure. These results demonstrate that DE2 thin film is a candidate for a photocarrier-generating material in organic solar cells.

*Corresponding author. Address: Institut für Angewandte Physik, Universität Tübingen, Auf der Morgenstelle 10, 72076 Tübingen, Germany, Fax: +49-7071 29 5110. Email address: t.hosokai.jp@gmail.com (T. Hosokai) 


\section{Introduction}

Organic photovoltaic cells (OPVs) have attracted considerable attention because of their potential use in low-cost solar energy conversion, colourful and sophisticated design and device flexibility [1-8]. In OPVs, organic thin films sandwiched between a transparent conductive substrate and back electrode play a role in photocarrier generation. A strong extinction coefficient in a wide optical range, especially in the visible range, is essential for organic thin films to achieve high photocarrier generation efficiency. Optical properties of organic molecules, such as the optical gap and extinction coefficient, can be tuned by designing the chemical structures and/or substituents. However, many significant differences are often observed unexpectedly between the optical spectra of organic thin films and the spectrum of a single molecule due to the coupling of molecular excitons among long-range molecules, molecular distortion, defects in a solid and so on [9-11]. Such difference between a solid and the component molecule is sometimes associated with an excellent materials property, and an organic photoconductor is a successful example. In this context, understanding the optical properties of organic thin films is potentially of important for the promotion of OPVs as well as being of fundamental interest.

$N, N^{\prime}$-bis[4-( $N, N$-diethylamino)benzylidene]diaminomaleonitrile (DE2), shown in Figure 1, is a bisazomethine dye derived from diaminomaleonitrile together with 4-diethlaminobenzaldehyde and has a high extinction coefficient of $1.3 \times 10^{5} \mathrm{dm}^{3} \mathrm{~mol}^{-1} \mathrm{~cm}^{-1}$ at $546 \mathrm{~nm}$ in chloroform [12]. Because of its red fluorescence in solution and in the solid state, DE2 has been examined as a fluorescent material for organic light-emitting diodes [13]. On the other hand, Matsumoto et al. reported that DE2 forms Jaggregates in vacuum-deposited thin films of a specific thickness [14]. J-aggregates are well known as photosensitizers in silver halide films because of their sharp and narrow absorption band (J-band) [15]. In addition to the above-mentioned properties, the large oscillator strength and fast electronic response of J-aggregates have attracted attention from the viewpoint of applications in various optoelectronic devices including OPVs [15-18]. However, the stability of conventional J-aggregates composed of an ionic dye in thin films remains a practical problem. DE2 is a non-ionic dye, and thus, its J-aggregate 
thin films are extremely stable. The absorption spectra of the J-aggregate thin films do not significantly change even after preservation for one year in ambient conditions or after thermal treatment at $373 \mathrm{~K}$ for $1 \mathrm{~h} \mathrm{[14].}$

These points of interest have stimulated our study of DE2 thin films. To date, we have reported the molecular arrangement in a single crystal, surface morphology and the electronic structure and photoluminescence properties of DE2 thin films in detail [19-22]. In the present work, we study the photovoltaic properties of DE2 thin films forming J-aggregates in Schottky-type cells. The simplicity of the device structure (i.e., electrode-organic thin film-electrode) makes it a good starting point for evaluating the photovoltaic properties of organic materials. We show that the DE2 thin films have ptype semiconductor properties and can be applied in Schottky-type solar cells. In addition, it is noted that the incident photon to collected electron efficiency (IPCE) is high and comparable to that reported for a similar primitive device structure with metal phthalocyanine thin films, suggesting that DE2 thin films are strong candidates for photocarrier-generating materials in OPVs.

\section{Experiment}

DE2 was synthesized according to the literature [23] and purified using the recrystallization method. Photovoltaic cells were fabricated on indium tin oxide (ITO) substrates to produce a common sandwich structure. After cleaning the ITO substrates ultrasonically with pure water, 2-propanol, acetone and chloroform for 15 min consecutively and blow-drying them in nitrogen, the DE2 thin films were prepared by vacuum deposition at a pressure of $3 \times 10^{-4} \mathrm{~Pa}$. The film thickness $(151 \mathrm{~nm})$ was then determined using a surface profiler (Dektak IIA, Sloan). We note that the film thickness gives rise to the formation of J-aggregates, as we reported previously [14]. Finally, a semi-transparent Al electrode $\left(0.28 \mathrm{~cm}^{2}\right)$ was prepared using a metal shadow mask on the DE2 thin film by vacuum deposition.

We used a Keithley 6487 unit to measure the current-voltage (I-V) characteristics and shortcircuit photocurrent. The light source was intensity-controlled monochromatic light from a tungsten lamp with a monochromator (CT-10, Jasco). Devices were illuminated through either the ITO electrode 
or $\mathrm{Al}$ electrode at an intensity of $5 \mu \mathrm{W} \mathrm{cm}{ }^{-2}$ to determine the IPCE spectra and photo $I-V$ curve, where the transmittance of the $\mathrm{Al}$ electrode was taken into account so that the intensity at the surface of the

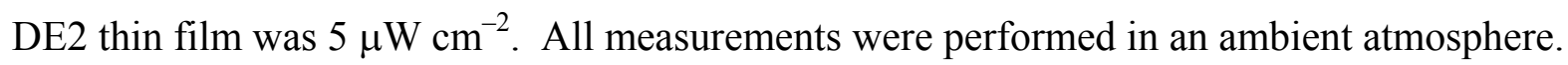

He I ultraviolet photoelectron spectroscopy (UPS) was carried out using ESCALAB (VG Scientific Ltd.). DE2 thin films with various thicknesses were prepared on a cleaned ITO substrate by the same procedure as that mentioned above. After breaking the vacuum, each sample was transferred immediately into the UPS apparatus. The samples were biased at $-5 \mathrm{~V}$ during the measurement to obtain the cut-off position of UPS spectra.

\section{Results and Discussion}

\subsection{Electronic structure of DE2 thin film on ITO}




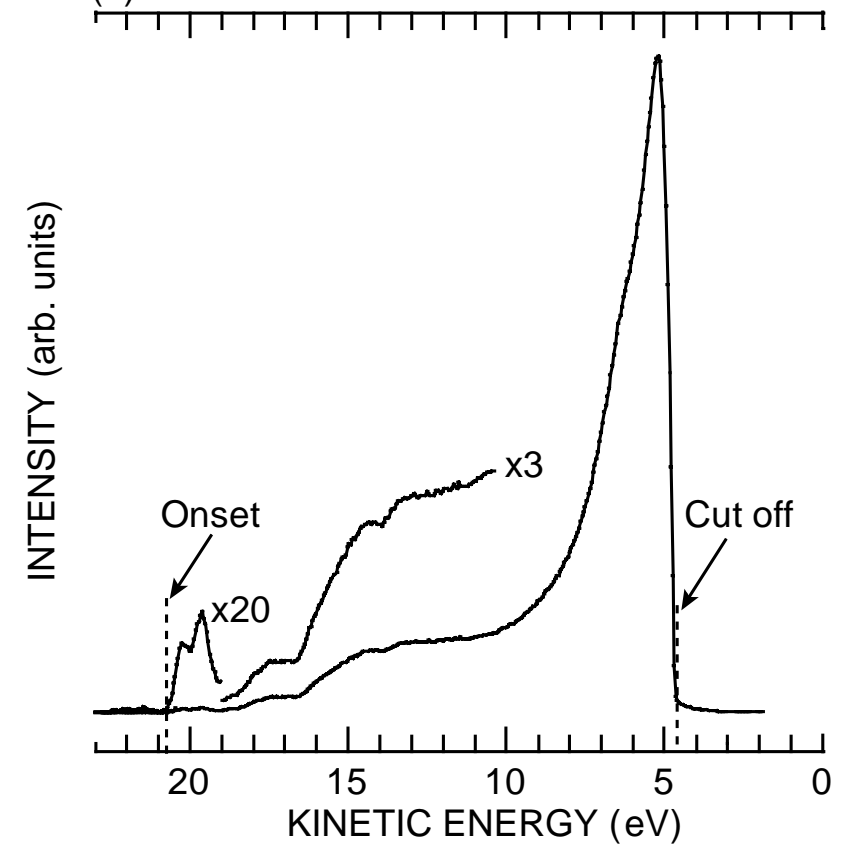

(b) Energy level diagram

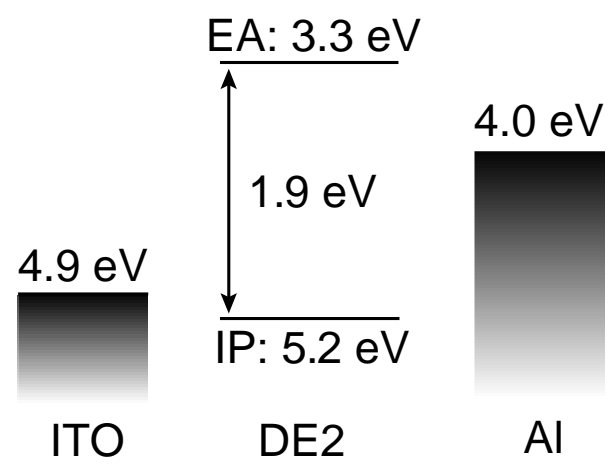

Figure 2 (a) shows the typical UPS spectrum of DE2 thin films on an ITO substrate. The ionization potential (IP) of DE2 thin films was determined to be $5.2 \mathrm{eV}$ by subtracting the energy width of the entire spectrum, which is the energy range from the onset to the cut-off position indicated in Fig. 2 (a), from the excitation source energy of $21.2 \mathrm{eV}$. Fig. 2 (b) shows an energy level diagram of DE2 thin films, where a measured work function (WF) of the ITO and a typical WF of Al electrode [24] are compared. The electron affinity (EA) of $3.3 \mathrm{eV}$ was obtained by subtracting the optical band gap of $1.9 \mathrm{eV}$ for DE2 thin film from the IP. According to the work by Kahn et al. [24], the IP and EA of DE2 thin films are comparable to those of hexadecahydro metal phthalocyanine thin films, which are typical p-type organic semiconductors used for OPVs. This could imply a p-type semiconductor property for the DE2 thin films [24]. 
It is worth noting that the UPS spectra determined here are quite similar to those determined by Hosokai et al. [20] despite the preparation conditions, except substrate temperature, being significantly different; i.e., in-situ preparation under ultrahigh vacuum condition with very slow evaporation rate (0.2-0.3 $\AA / \mathrm{min})$. This suggests that the molecular orientation of DE2 thin films is similar for the two deposition conditions and the molecules lie nearly flat on ITO substrates independent of the film thickness [20].

\subsection{Characterization of semiconducting properties and photocarrier generation of DE2 thin film}

Figure 3 shows the dark $I-V$ characteristic for the ITO/DE2/Al device. The dark current significantly increased at $\sim 1 \mathrm{~V}$ with forward bias, whereas the current did not change as greatly with reverse bias. This clear rectification behavior demonstrates the semiconductor property of DE2 thin films, which is presumably due to the Schottky barrier between DE2 and the Al electrode, considering the photocurrent action spectra presented below. 


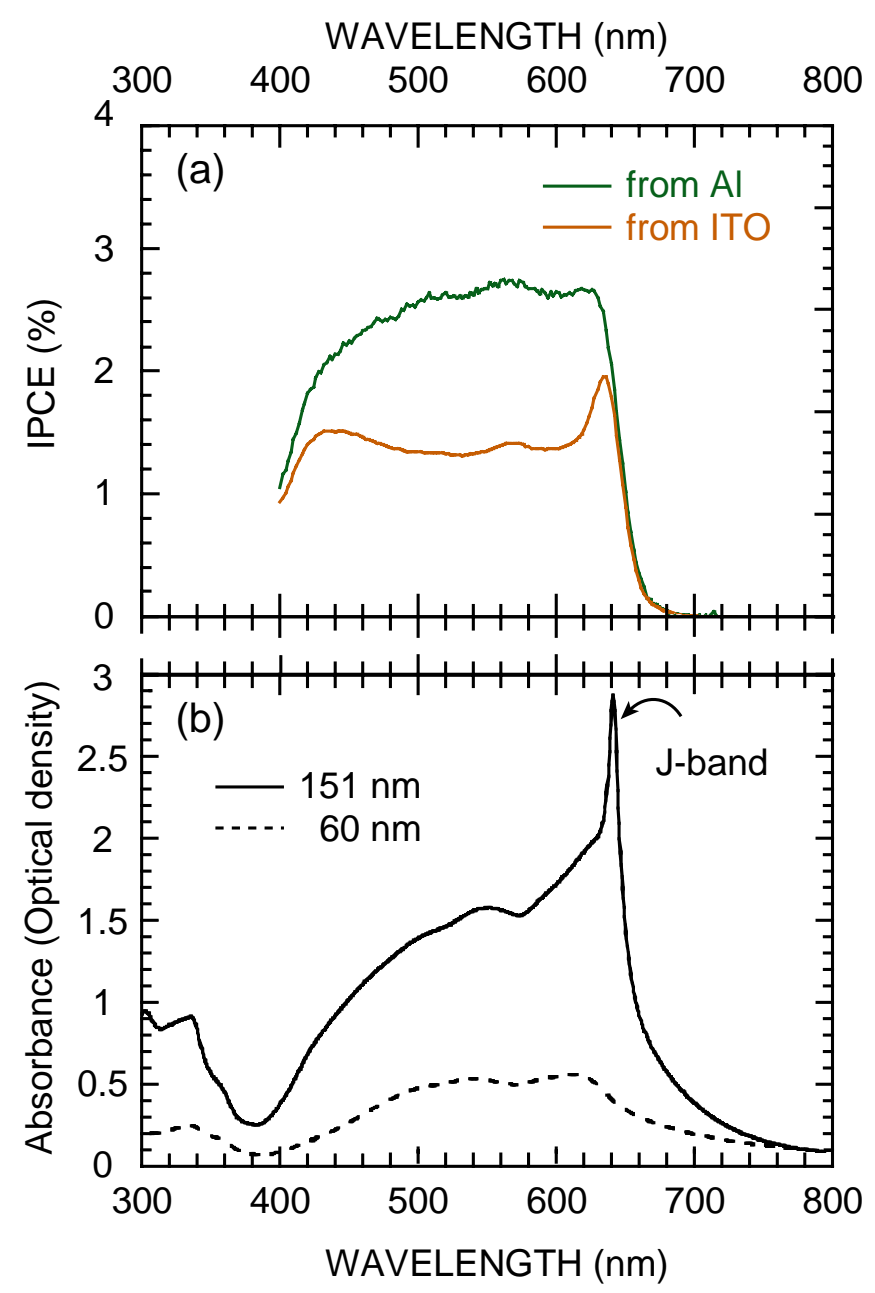

We determined the short-circuit photocurrent action spectra for the ITO/DE2/A1 device and calculated the external quantum efficiency (i.e., the IPCE) as

$\operatorname{IPCE}[\%]=124 \times\left|J_{s c}\right| /\left(\lambda \times I_{\mathrm{p}}\right)$,

where $J_{s c}\left[\mu \mathrm{A} \mathrm{cm}^{-2}\right]$ is the short-circuit photocurrent density and $\lambda[\mathrm{nm}]$ and $I_{\mathrm{p}}\left[\mathrm{mW} \mathrm{cm}^{-2}\right]$ are the wavelength and intensity of the incident light, respectively. Figure 4 (a) shows the IPCE spectra of ITO/DE2/Al under illumination through the Al and ITO electrodes (IPCE $\mathrm{Al}_{\mathrm{Al}}$ and IPCE $\mathrm{ITO}_{\mathrm{ITO}}$ spectra). For a comparison of the IPCE spectra, absorption spectra of DE2 thin films with a thickness of $151 \mathrm{~nm}$ and $60 \mathrm{~nm}$ are shown in Fig 4 (b). As reported previously [14], the sharp peak at $641 \mathrm{~nm}$ in the absorption spectrum of the $151 \mathrm{~nm}$-thick film has been characterized as the J-band, while the spectrum of the $60 \mathrm{~nm}$-thick film has no such a J-band, showing there is no J-aggregates in the 
film. Short-circuit photocurrent was observed in a primitive device structure of ITO/DE2/Al. This demonstrates that DE2 thin films can generate and transport photocarriers in photovoltaic cells.

IPCE spectra were dependent on the direction of illumination. We observed higher IPCE throughout the measurement region by illuminating through the $\mathrm{Al}$ electrode. This result was valid even if a small adsorption of a visible light of the ITO was taken into account for the IPCE ITO spectrum. These results suggest that a Schottky barrier is formed at the interface between DE2 and the $\mathrm{Al}$ electrode. We note that the IPCE of a few percent in the $\operatorname{IPCE}_{\mathrm{Al}}$ spectrum (e.g., 2.7\% at $564 \mathrm{~nm}$ ) is comparable to that for traditional photovoltaic cells with metal phthalocyanine thin films in a similar primitive device structure $[25,26]$.

In general, the Schottky barrier region in Schottky-type OPVs is thought to be limited to the interface between organic thin films and an electrode or within a thickness of several monolayers from the interface. Therefore, IPCE depends on the number of incident photons (or excitons) reaching to the Schottky barrier region through the organic thin films or an electrode. For a case of the DE2 device structure, under illumination through the Al electrode incident photons are absorbed at the interface (i.e., the Schottky barrier region), and thus, photocarriers are efficiently generated. On the other hand, under illumination through the ITO electrode, photocarrier generation is limited because quite less incident photons can reach to the DE2/Al interface in the measurement region of the IPCE spectra due to the strong absorption of the DE2 thin film (see Fig. 4 (b)). This phenomenon results in the different spectral shape for IPCE $_{\mathrm{Al}}$ and IPCE $\mathrm{ITO}_{\mathrm{IT}}$ spectra and is the so-called filtering effect that is often observed in Schottky-type photovoltaic cells [27-30]. As a consequence, the IPCE $\mathrm{Al}_{\mathrm{Al}}$ spectrum matches the absorption spectrum (the symbatic response) except for the J-band at $641 \mathrm{~nm}$, whereas the IPCE ITO spectrum is more concave (the antibatic response). Although the reason why no clear contribution of the J-band was observed in the IPCE spectra has not yet been clarified, the excitons of the J-aggregates might not contribute to the generation of photocarriers in this device configuration or the density of J-aggregates might be very low. Although one might suspect that the peak at $640 \mathrm{~nm}$ in the IPCE $\mathrm{ITO}_{\text {ITO }}$ spectra could be assigned to the contribution of the J-band, we confirmed 
that the peak is derived from the filtering effect by comparing the absorption spectra of the Jaggregate thin film and the non J-aggregate thin film shown in Fig. 4(b) and the IPCE spectra [31]. It is worth noting that a previous study showed that excited J-aggregates transfer energy to the metal electrode efficiently through the excitation of surface plasmon, and thus, the contribution of Jaggregates to the IPCE appears to decrease [32]. Such non-radiative decay of excited J-aggregates can be prevented by the insertion of a $\mathrm{C}_{60}$ layer between J-aggregates and the metal electrode [16]. To prevent the non-radiative decay, we also constructed heterojunction devices of ITO/DE2/C $60 / \mathrm{Al}$ and determined the IPCE spectra. The IPCE increased for both irradiation conditions and showed maximally $14.0 \%$ at $548 \mathrm{~nm}$ for IPCE $_{\mathrm{Al}}$ spectrum because of an efficient charge separation at the donar (DE2) / acceptor $\left(\mathbf{C}_{60}\right)$ interface. However, a clear contribution of the J-band was not observed in the IPCE spectra. These results for the heterojunction devices will be discussed in detail elsewhere.

\subsection{Characterization of photovoltaic properties of DE2 thin film}

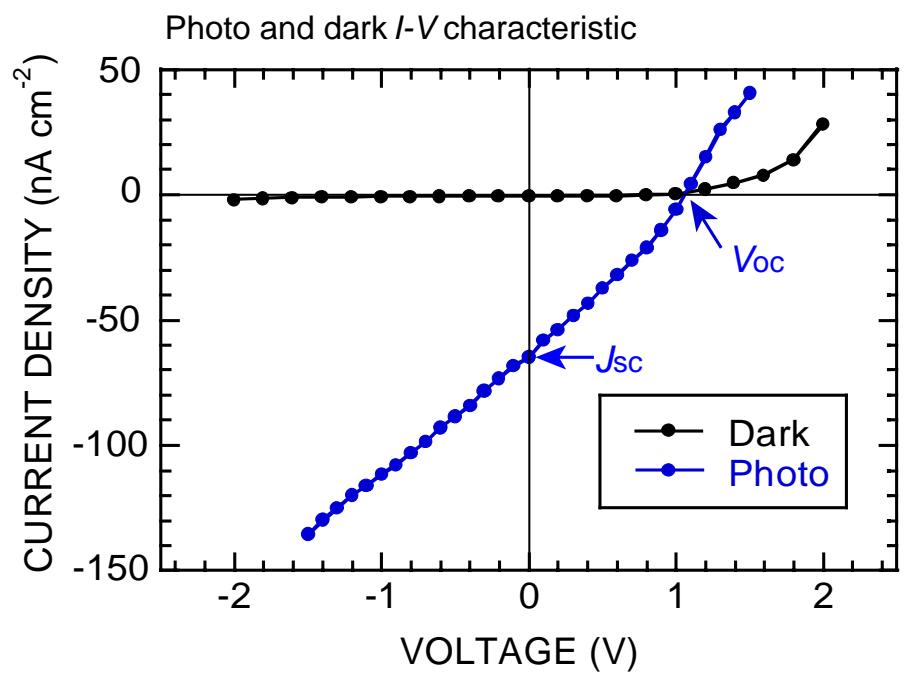

Figure 5 shows the photo $I-V$ characteristic of ITO/DE2/Al illuminated with $600 \mathrm{~nm}$ monochromatic light through the Al electrode, where the dark $I-V$ characteristic depicted in Fig. 3 is also shown for a comparison. Several fundamental parameters such as the open circuit voltage $\left(V_{\mathrm{oc}}\right)$, fill factor $(F F)$ and $J_{\mathrm{sc}}$ can be extracted from the photo $I-V$ curve. $F F$ is defined as the maximum power output of the cell divided by the product of $V_{\mathrm{oc}}$ and $J_{\mathrm{sc}}$. Under this measurement condition, we obtained $V_{\mathrm{oc}}=1.06 \mathrm{~V}$, $J_{\mathrm{sc}}=-6.47 \times 10^{-5} \mathrm{~mA} \mathrm{~cm}^{-2}$ and $F F=0.28$. The observed value of $V_{\mathrm{oc}}$ is slightly higher than that for a 
metal phthalocyanine thin film having the same device structure [25, 26, and 33]. A similar value of FF is frequently obtained for Schottky-type OPVs due to high series resistance (Rs) of organic thin films [34, 35]. For the Photo $I-V$ curve, the higher current density above the $V_{\text {oc }}$ and the continuous change in the current in the third quadrant can be ascribed to the decreasing of the $R s$ due to the photoconductivity of the DE2 thin film by light illumination.

The power conversion efficiency of a photovoltaic cell $(\eta)$ is given by $\eta(\%)=\left(\left|J_{\mathrm{sc}} V_{\mathrm{oc}}\right| / I_{O} \times F F\right) \times 100$,

where $I_{O}=5 \mu \mathrm{W} \mathrm{cm}^{-2}$ is the incident light power in this measurement. The efficiency $\eta$ was estimated to be $0.38 \%$.

In general, the efficiency $\eta$ of a Schottky-type OPV depends largely on the intensity of the incident light, surface treatment of the substrate, cell area and other considerations. Nevertheless, the observed efficiency $\eta$ of the DE2 cell is comparable to values for ITO/metal phthalocyanine/Al devices reported in the literature $[25,26]$, for which the measurement conditions were similar to those in this study. Although optimization of the DE2 device possibly improves the efficiency $\eta$ [33], the observed efficiency $\eta$ indicates that DE2 thin films can be used in OPVs as an active layer.

\section{Conclusions}

We studied the photovoltaic properties of DE2 thin films forming J-aggregates in a Schottky-type cell. It was revealed that the DE2 thin films form a Schottky barrier at the interface with the Al electrode and work as a p-type semiconductor. Although we did not find an apparent contribution of J-aggregates in the photocurrent action spectra, we observed IPCE comparable to that of metal phthalocyanine thin films. These results indicate that ITO/DE2/Al devices can be applied in Schottky-type solar cells and that a DE2 thin film is a candidate for a photocarrier-generating material in OPVs.

\section{Acknowledgements}


Part of this work was supported by the Industrial Research Grant Program of the New Energy and Industrial Technology Development Organization (NEDO) of Japan. The authors are grateful to Dr. T. Tanaka (Sumitomo Chemical Co. Ltd.) for helpful discussions.

\section{References}

[1] C.W. Tang, Appl. Phys. Lett. 48 (1986) 183.

[2] G. Yu, J. Gao, J. C. Hummelen, F. Wudl, A. J. Heeger, Science 270 (1995) 1789.

[3] J. Xue, S. Uchida, B. P. Rand, S. R. Forrest, Appl. Phys. Lett. 84 (2004) 3031.

[4] S. Günes, H. Neugebauer, N. S. Sariciftci, Chem. Rev. 107 (2007) 1324.

[5] C. J. Brabec, Sol. Energy Mater. Sol. Cells 83 (2004) 273.

[6] B. P. Rand, J. Genoe, P. Heremans, J. Poortmans, Prog. Photovolt: Res. Appl. 15 (2007) 659.

[7] K. Suemori, T. Miyata, M. Yokoyama, M. Hiramoto, Appl. Phys. Lett. 85 (2004) 6269.

[8] T. Taima, M. Chikamatsu, Y. Yoshida, K. Saito, K. Yase, Appl. Phys. Lett. 85 (2004) 6412.

[9] M. Kasha, H. R. Rawls, M. A. El-Bayoumi, Pure Appl. Chem. 11 (1965) 371.

[10] J. Mizuguchi, G. Rihs, H. R. Karfunkel, J. Phys. Chem. 99 (1995) 16217.

[11] U. Heinemeyer, R. Sholz, L. Gisslén, M. I. Alonso, J. O. Ossó, M. Garriga, A. Hinderhofer, M. Kytka, S. Kowarik, A. Gerlach, F. Schreiber, Phys. Rev. B 78 (2008) 085210.

[12] R. W. Begland, Preparation of disperse bisanil dyes derived from diaminomaleonitrile, US Patent $3962220,1976$.

[13] J. Yu, Z. Chen, M. Sone, S. Miyata, M. Li, T. Watanabe, Jpn. J. Appl. Phys. 40 (2001) 3201.

[14] S. Matsumoto, T. Kobayashi, T. Aoyama, T. Wada, Chem. Comm. (2003) 1910.

[15] T. Kobayashi, J-Aggregates, World Scientific Publishing Co. Pte. Ltd., 1996.

[16] K. Saito, Jpn. J. Appl. Phys. 38 (1999) L1140.

[17] M. Kawasaki, S. Aoyama, Chem. Comm. (2004) 988.

[18] M. Era, C. Adachi, T. Tsutsui, S. Saito, Chem. Phys. Lett. 178 (1991) 488. 
[19] S. Matsumoto, K. Shirai, K. Kobayashi, T. Wada, M. Shiro, Zeitschrift für Kristallographie 219 (2004) 239.

[20] T. Hosokai, S. Kera, S. Matsumoto, K. K. Okudairam N. Ueno, Mol. Cryst. Liq. Cryst. 472 (2007) 43.

[21] T. Kobayashi, S. Masumoto, T. Tanaka, H. Kunugita, K. Ema, T. Aoyama, T. Wada, Phys. Chem. Chem. Phys. 7 (2005) 1726.

[22] S. Matsumoto, M. Satoh, D. Tsuchida, T. Kobayashi, T. Aoyama, T. Wada, Transactions of MRS-J, $30(2005) 345$.

[23] K. Shirai, M. Matsuoka, K. Fukunishi, Dyes Pigm. 47 (2000) 107.

[24] A. Kahn, N. Koch, W. Gao, J. Polym. Sci. Polym. Phys. 41 (2003) 2529.

[25] T. Iwase, Y. Haga, Jpn. J. Appl. Phys. Part 142 (2003) 5330.

[26] H. Yonehara, C. Pac, Thin Solid Films 278 (1996) 108.

[27] R. O. Loutfy, J. H. Sharp, C. K. Hisao, R. Ho, J. Appl. Phys. 52 (1981) 5218.

[28] A. K. Ghosh, T. Feng, J. Appl. Phys. 49 (1978) 5982.

[29] M. G. Harrison, J. Grüner, G. C. Spencer, Phys. Rev. B 55 (1997) 7831.

[30] T. Stübinger, W. Brütting, J. Appl. Phys., 90 (2001) 3632.

[31] It has been considered by a thickness dependence of optical spectra [14] and a study of surface morphology [22] that the J-aggregates of DE2 molecules is existing at a surface but not in a bulk region of the films. According to Ghosh and Feng [28], an absorption spectrum of a bulk region which induces a filtering effect in photocurrent action spectra can be approximately calculated by a following relation, IPCE $_{\mathrm{Al}} / \mathrm{IPCE}_{\mathrm{ITO}}$. For the case of our Schottky cell, the calculated absorption spectrum does not match the absorption spectrum of the J-aggregate thin film (thickness: $151 \mathrm{~nm}$ ) but can match the non J-aggregate thin film shown (thickness: $60 \mathrm{~nm}$ ) in

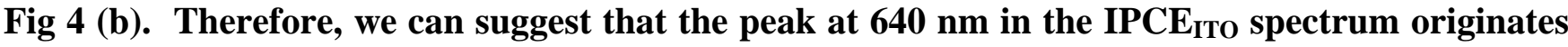
from the filtering effect due to the adsorption in the bulk region of DE2 thin films without Jaggregates. 
[32] K. Saito, J. Phys. Chem. B 103 (1999) 6579.

[33] C. Y. Kwong, A. B. Djurišiĉ, P. C. Chui, L. S. M. Lam, W. K. Chan, Appl. Phys. A 77 (2003) 555 and references therein.

[34] T. Aernouts, W. Geens, J. Poortmans, P. Heremans, S. Borghs, R. Mertens, Thin Solid Films, 404-403 (2002) 297.

[35] S. Uchida, J. Xue, B. P. Rand, S. R. Forrest, Appl. Phys. Lett., 84 (2004) 4218. 
Figure 1. Chemical structure of DE2.

Figure 2. (a) UPS spectrum of DE2 thin films (thickness: $17 \mathrm{~nm}$ ) on ITO substrate. (b) Energy level diagram of ITO/DE2/Al.

Figure 3. Dark $I-V$ characteristic of ITO/DE2 $(151 \mathrm{~nm}) / \mathrm{Al}$. The forward bias corresponds to the Al electrode being negative.

Figure 4. (a) IPCE spectra of ITO/DE2 (151 nm)/Al illuminated through the Al or ITO electrode. (b) Absorption spectra of the DE2 thin films with a thickness of $151 \mathrm{~nm}$ and $60 \mathrm{~nm}$.

Figure 5. Photo and dark $I-V$ characteristic of ITO/DE2 (151 nm)/Al. The photo $I-V$ characteristic was determined by illuminating a monochromatic light of $600 \mathrm{~nm}$ with an intensity of $5 \mu \mathrm{W} \mathrm{cm} \mathrm{cm}^{-2}$ at $\mathrm{DE} / \mathrm{Al}$ interface through the $\mathrm{Al}$ electrode. 